\title{
Macrophage Infiltration Is a Causative Factor for Ligamentum Flavum Hypertrophy through the Activation of Collagen Production in Fibroblasts
}

Takeyuki Saito, ${ }^{* \dagger}$ Masamitsu Hara, ${ }^{* \dagger}$ Hiromi Kumamaru, ${ }^{\dagger}$ Kazu Kobayakawa, ${ }^{\dagger}$ Kazuya Yokota, ${ }^{\ddagger}$ Ken Kijima, ${ }^{* \dagger}$

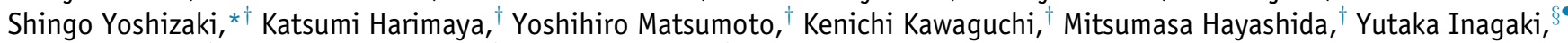
Keiichiro Shiba, ${ }^{\ddagger}$ Yasuharu Nakashima, $^{\dagger}$ and Seiji Okada* ${ }^{\star \dagger}$

From the Departments of Advanced Medical Initiatives* and Orthopaedic Surgery, ${ }^{\dagger}$ Graduate School of Medical Sciences, Kyushu University, Fukuoka, Japan; the Department of Orthopaedic Surgery, ${ }^{\ddagger}$ Spinal Injuries Center, Fukuoka, Japan; the Center for Matrix Biology and Medicine, ${ }^{\S}$ Graduate School of Medicine, and the Department of Regenerative Medicine, "School of Medicine, Tokai University, Isehara, Japan

\author{
Accepted for publication \\ August 15, 2017. \\ Address correspondence to Seiji \\ Okada, M.D., Ph.D., Depart- \\ ment of Orthopaedic Surgery, \\ Graduate School of Medical \\ Sciences, Kyushu University, \\ 3-1-1 Maidashi, Higashi-ku, \\ Fukuoka 812-8582, Japan. \\ E-mail: seokada@ortho.med. \\ kyushu-u.ac.jp.
}

\begin{abstract}
Ligamentum flavum (LF) hypertrophy causes lumbar spinal canal stenosis, leading to leg pain and disability in activities of daily living in elderly individuals. Although previous studies have been performed on LF hypertrophy, its pathomechanisms have not been fully elucidated. In this study, we demonstrated that infiltrating macrophages were a causative factor for LF hypertrophy. Induction of macrophages into the mouse LF by applying a microinjury resulted in LF hypertrophy along with collagen accumulation and fibroblasts proliferation at the injured site, which were very similar to the characteristics observed in the severely hypertrophied LF of human. However, we found that macrophage depletion by injecting clodronate-containing liposomes counteracted LF hypertrophy even with microinjury. For identification of fibroblasts in the LF, we used collagen type I $\alpha_{2}$ linked to green fluorescent protein transgenic mice and selectively isolated green fluorescent protein-positive fibroblasts from the microinjured LF using laser microdissection. A quantitative RT-PCR on laser microdissection samples revealed that the gene expression of collagen markedly increased in the fibroblasts at the injured site with infiltrating macrophages compared with the uninjured location. These results suggested that macrophage infiltration was crucial for LF hypertrophy by stimulating collagen production in fibroblasts, providing better understanding of the pathophysiology of LF hypertrophy. (Am J Pathol 2017, 187: 2831-2840; https://doi.org/10.1016/j.ajpath.2017.08.020)
\end{abstract}

Lumbar spinal canal stenosis (LSCS) is a common spinal disorder in elderly individuals and leads to severe disability in activities of daily living attributable to lower back pain, leg pain, and claudication. ${ }^{1}$ Ligamentum flavum (LF) is a spinal ligament that covers a large part of the posterior and lateral walls of the spinal canal (Figure 1, A-C). Thus, hypertrophy of the LF is a major cause of LSCS by compressing the nerve roots and/or cauda equine $^{2}$ (Figure 1, D-F). There have been pathologic studies on LF hypertrophy using human samples. In the hypertrophied LF, the loss of elastic fibers, an excessive accumulation of collagen fibers, ${ }^{3,4}$ the infiltration of inflammatory cells, ${ }^{5}$ and an increased expression of several fibrosis-related factors, such as transforming growth factor (TGF)- $\beta 1$ and connective tissue growth factor, 6,7 were observed. However, the pathomechanisms of LF hypertrophy have not been fully elucidated in part because of the lack of animal studies.

In the hypertrophied LF of patients with LSCS, as in other fibrotic diseases that affect organs, excessive collagen synthesis is a pathologic characteristic, ${ }^{8}$ and the activation of fibroblasts, which are known to produce collagen, is considered to play a role in the disease process. ${ }^{9}$ However, the in vivo role of fibroblasts in LF hypertrophy remains elusive because of a lack of specific markers. Although previous studies using human tissue

Supported in part by grants-in-aid for Scientific Research $16 \mathrm{H} 05450$ and Challenging Exploratory Research 16K15668 from the Ministry of Education, Science, Sports and Culture of Japan (S.O.).

Disclosures: None declared. 

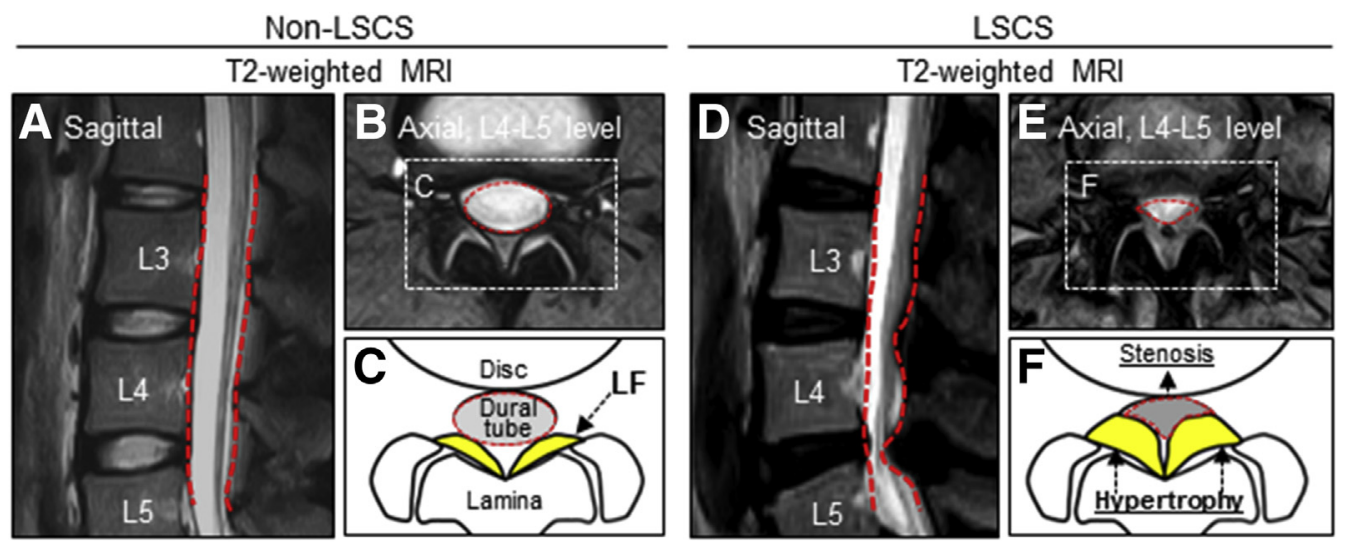

Figure 1 Magnetic resonance imaging (MRI) and schematic illustrations of human lumbar spine. A and B: Sagittal and axial T2-weighted MRI showing the nonhypertrophied ligamentum flavum (LF) of patients without lumbar spinal canal stenosis (LSCS). C: A schematic illustration of the boxed area in B. D and E: Sagittal and axial T2-weighted MRI showing the hypertrophied LF of patients with LSCS. F: A schematic illustration of the boxed area in E. The dashed red lines indicate the outline of the dural tube. L4, fourth lumbar vertebra; L5, fifth lumbar vertebra.

cultures have attempted to investigate the function of LF fibroblasts, the in vitro cultures comprised not only many fibroblastlike cells but also various types of cells, including chondrocytes and osteoblasts. ${ }^{7,10}$ Thus, a method of selectively identifying fibroblasts is necessary to clarify their properties in the disease process of LF hypertrophy.

In addition to fibroblasts, macrophages have been reported to contribute to fibrotic diseases in several organs. For instance, macrophages secrete cytokines and growth factors, such as TGF- $\beta 1$, leading to enhanced collagen production in fibroblasts and the deterioration of fibrosis. ${ }^{11-13}$ Macrophage infiltration and the TGF- $\beta 1$ expression have been reported to also occur in the hypertrophied $\mathrm{LF}^{5}$, suggesting that macrophages are associated with the process of LF hypertrophy. However, whether they are causative or merely a consequence of LF hypertrophy remains unclear because human samples from patients with LSCS patients had advanced histologic changes, including excessive collagen accumulation.

In the present study, to investigate the interplay between fibroblasts and macrophages on LF hypertrophy, we used collagen type I $\alpha_{2}$ linked to green fluorescent protein (COL1a2-GFP) transgenic mice and examined the involvement of GFP-positive fibroblasts in the disease process of LF hypertrophy in vivo. In addition, we induced macrophage infiltration in the mouse LF and investigated the influence on fibroblasts.

\section{Materials and Methods}

\section{The Human LF}

Human samples were collected at surgery from 7 male and 8 female patients with LSCS (mean age, 77.7 years; range, 71 to 86 years) as the severely hypertrophied $\mathrm{LF}$ ( $\geq 4 \mathrm{~mm}$ ) and 16 male and 14 female patients with lumbar disk herniation (mean age, 39.3 years; range, 28 to 62 years) as the nonhypertrophied $\mathrm{LF}(<2 \mathrm{~mm})$ and mildly hypertrophied $\mathrm{LF}(\geq 2 \mathrm{~mm})(n=15$ per group) (Table 1). To measure the LF thickness, axial T1weighted magnetic resonance imaging was performed before surgery. ${ }^{14}$ For the histologic analysis, half of each LF was fixed in $4 \%$ paraformaldehyde and dehydrated in sucrose solution. The LF was embedded in OCT compound, frozen in liquid nitrogen, and cut into $10-\mu \mathrm{m}$ sections using a cryostat. The other half was used for a quantitative RT-PCR (RTqPCR). All procedures were approved by the Institutional Review Board of Kyushu University.

Table 1 Demographic Data of Human Samples

\begin{tabular}{lccc}
\hline Variable & Nonhypertrophied LF & Mildly hypertrophied LF & Severely hypertrophied LF \\
\hline $\begin{array}{l}\text { Age, means } \pm \text { SEM (range), y } \\
\text { Sex, no. }\end{array} \quad 30.6 \pm 1.2(28$ to 40) & $48.1 \pm 1.5(42$ to 62) & $77.7 \pm 1.4(71$ to 86$)$ \\
$\quad$ Male & 10 & 8 & 6 \\
$\quad$ Female & 5 & 7 & 9 \\
Diagnosis of patients & LDH & & LSCS \\
Level of harvested LF, no. & 3 & 6 & 4 \\
$\quad$ L3/4 & 6 & 8 & 10 \\
L4/5 & 6 & 1 & 1 \\
L5/S & $1.73 \pm 0.04$ & $3.01 \pm 0.09$ & $5.102 \pm 0.11$ \\
LF thickness, means \pm SEM mm & & & 4 \\
\hline
\end{tabular}

LF, ligamentum flavum; LDH, lumbar disk herniation; LSCS, lumbar spinal canal stenosis. 
Table 2 Primers Used for Quantitative RT-PCR

\begin{tabular}{|c|c|}
\hline Gene (accessi & Primer \\
\hline \multicolumn{2}{|c|}{ Human } \\
\hline \multicolumn{2}{|c|}{ COL1A1 (NM_000088.3) } \\
\hline Forward & 5'-TCCCATGGCTCTTGCAACATCTCC-3' \\
\hline Reverse & 5'-CACAAGGAACAGAACAGTCTCTCC-3' \\
\hline \multicolumn{2}{|c|}{ COL1A2 (NM_001901.2) } \\
\hline Forward & 5'-ACAGTTTCATTAACTCCTTCCССC-3' \\
\hline Reverse & 5'-TGGGTTTTAAACTTCCСTCTGTGG-3' \\
\hline \multicolumn{2}{|c|}{ COL3A1 (NM_000090.3) } \\
\hline Forward & $5^{\prime}$-АTCAACTGCTTGTAAAGGTGCTCC- $3^{\prime}$ \\
\hline Reverse & $5^{\prime}$-GGGAGACATGTACAGATTTTGTGC- $3^{\prime}$ \\
\hline \multicolumn{2}{|c|}{ TGF- $\beta 1$ (NM_000660.6) } \\
\hline Forward & $5^{\prime}$-ATGGGGGCTGTATTTAAGGACACC- $3^{\prime}$ \\
\hline Reverse & $5^{\prime}$-AAAGGAATAGTGCAGACAGGCAGG- $3^{\prime}$ \\
\hline \multicolumn{2}{|c|}{ CTGF (NM_001901.2) } \\
\hline Forward & $5^{\prime}$-АTTTTAGCGTGCTCACTGACCTGC-3' \\
\hline Reverse & 5'-TCCAGTCTAATCGACAGGATTCCG-3' \\
\hline \multicolumn{2}{|c|}{ PDGFA (NM_002607.5) } \\
\hline Forward & 5'-TAAACTCGATGAGATGGAGGGTCG-3' \\
\hline Reverse & 5'-GGTTTCCAAGACATTCCTGCTTCC-3' \\
\hline \multicolumn{2}{|c|}{ GAPDH (NM_001256799.2) } \\
\hline Forward & $5^{\prime}$-GAGCACAAGAGGAAGAGAGAGACC- $3^{\prime}$ \\
\hline Reverse & 5'-TTGATGGTACATGACAAGGTGCGG- 3' \\
\hline \multicolumn{2}{|r|}{ 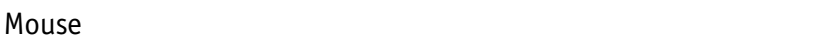 } \\
\hline \multicolumn{2}{|c|}{ Col1a1 (NM_007742.3) } \\
\hline Forward & $5^{\prime}-\mathrm{AACCCTGGAAACAGACGAACAACC-3^{ \prime }}$ \\
\hline Reverse & 5'-TGGTCACGTTCAGTTGGTCAAAGG-3' \\
\hline \multicolumn{2}{|c|}{ Col1a2 (NM_007743.2) } \\
\hline Forward & $5^{\prime}$-АТССААСТАAGTCTCСТСССТTGG-3' \\
\hline Reverse & $5^{\prime}$-CTCTGTGGAAGATAGTCAGAAGCC- $3^{\prime}$ \\
\hline \multicolumn{2}{|c|}{ Col3a1 (NM_009930.2) } \\
\hline Forward & 5'-TAAAGAAGTCTCTGAAGCTGATGG- $3^{\prime}$ \\
\hline Reverse & 5'-ATCTATGATGGGTAGTCTCATTGC-3' \\
\hline \multicolumn{2}{|c|}{ Acta2 (encoding aSMA) (NM_007392.3) } \\
\hline Forward & 5'-CTCACTATGCACCTGGATCATTGC-3' \\
\hline Reverse & 5'-GTGTGAGAGTCTTGTGTACGATGC- $3^{\prime}$ \\
\hline \multicolumn{2}{|c|}{ Tgfb1 (NM_011577.1) } \\
\hline Forward & $5^{\prime}-\mathrm{TGGACACACAGTACAGCAAGGTCC}-3^{\prime}$ \\
\hline Reverse & 5'-ATCATGTTGGACAACTGCTCCACC-3' \\
\hline \multicolumn{2}{|c|}{ Pdgfa (NM_008808.3) } \\
\hline Forward & $5^{\prime}$-AGACAGATGTGAGGTGAGATGAGC- $3^{\prime}$ \\
\hline Reverse & $5^{\prime}-$ ACGGAGGAGAACAAAGACCGCACG-3' \\
\hline \multicolumn{2}{|c|}{ Tnfa (NM_013693.2) } \\
\hline Forward & 5'-TTATGGCTCAGGGTCCAACTCTGT-3' \\
\hline Reverse & 5'-TGGACATTCGAGGCTCCAGTGAAT- 3' \\
\hline \multicolumn{2}{|c|}{ Il1b (NM_013693.2) } \\
\hline Forward & $5^{\prime}$-GGGCTGGACTGTTTCTAATGCCTT-3' \\
\hline Reverse & 5'-CCATCAGAGGCAAGGAGGAAAACA- $3^{\prime}$ \\
\hline \multicolumn{2}{|c|}{ Il6 (NM_013693.2) } \\
\hline Forward & 5'-GCTCTCCTAACAGATAAGCTGGAG- $3^{\prime}$ \\
\hline Reverse & $5^{\prime}$-CCACAGTGAGGAATGTCCACAAAC- $3^{\prime}$ \\
\hline \multicolumn{2}{|c|}{ Ctgf (NM_010217.2) } \\
\hline Forward & 5'-GGCCATACAAGTAGTCTGTCAACC-3' \\
\hline Reverse & 5'-CACTCCAAAAAGTAGGCACACTGC- $3^{\prime}$ \\
\hline \multicolumn{2}{|c|}{ Gapdh (NM_008084.3) } \\
\hline Forward & 5'-GACTTCAACAGCAACTCCCACTCT-3' \\
\hline Reverse & 5'-GGTTTCTTACTCCTTGGAGGCCAT-3' \\
\hline
\end{tabular}

${ }^{*}$ Accession numbers are from the National Center for Biotechnology Information gene database (https://www.ncbi.nlm.nih.gov/gene).
Animals

Eight-week-old female C57BL/6 wild-type mice were used in this study (Japan SLC, Hamamatsu, Japan). COL1a2-GFP transgenic mice (C57BL/6 background) were generated and kindly donated by Dr. Yutaka Inagaki. ${ }^{15}$ As described previously, ${ }^{16}$ after the mice were transcardially fixed with $4 \%$ paraformaldehyde, the lumbar spine was removed and immersed in the same fixative. The spine was decalcified in EDTA and dehydrated in sucrose. The sample was then embedded in OCT compound, frozen in liquid nitrogen, and cut into $10-\mu \mathrm{m} \mathrm{sec-}$ tions on a cryostat. In all animal experiments, the mice were anesthetized intraperitoneally with an anesthetic mixture (medetomidine, $0.3 \mathrm{mg} / \mathrm{kg}$; midazolam, $4 \mathrm{mg} / \mathrm{kg}$; and butorphanol, $5 \mathrm{mg} / \mathrm{kg}$ ). ${ }^{17}$ The animal protocols were approved by the Committee of Ethics on Animal Experiment in the Faculty of Medicine, Kyushu University, in accordance with the Guidelines for Animal Experimentation.

\section{Histologic Analysis}

The sagittal sections of the human and mouse samples were subjected to hematoxylin and eosin (H\&E) and Elastica-van Gieson (EVG) staining, and the thickness of the mouse LF was measured ( $n=8$ per group). For immunostaining, the sections were stained with primary antibodies against Iba1 (1:200; rabbit; macrophage marker; Wako Pure Chemical Industries, Osaka, Japan) and GFP (1:200; pig; Frontier Institute, Hokkaido, Japan). The sections were then incubated with Alexa Fluor-conjugated secondary antibodies (1:200; Invitrogen, Carlsbad, CA). Nuclear counterstaining was performed using Hoechst 33342 (1:1000; Invitrogen). All images were obtained using a BZ-9000 digital microscope (Keyence, Osaka, Japan).

\section{RT-qPCR}

Total RNA was isolated from LF cells using an RNeasy Mini Kit (Qiagen, Hilden, Germany), and cDNA was synthesized from the total RNA using PrimeScript Reverse Transcriptase (Takara, Tokyo, Japan) according to the manufacturer's instructions. RTqPCR was performed using $20 \mu \mathrm{L}$ of reaction mixture with primers specific to the genes of interest (Table 2) and SYBR Premix Dimmer-Eraser (Takara). ${ }^{18}$ The mRNA levels in the human samples ( $n=15$ per group) and the mouse samples ( $n=8$ per group) were normalized to those of glyceraldehyde3-phosphate dehydrogenase mRNA. In the analysis of human samples, the severely hypertrophied LF specimens were divided into dorsal and dural layers as described previously. ${ }^{19}$ RT-qPCR was performed after obtaining samples (1 $\mathrm{mm}$ in width) from each layer of the severely hypertrophied LF and from the dural layer of the nonhypertrophied LF.

\section{Experimental Procedures}

To induce macrophage infiltration, after exposing the LF, a microinjury was applied to the dorsal layer of the mouse LF 
A
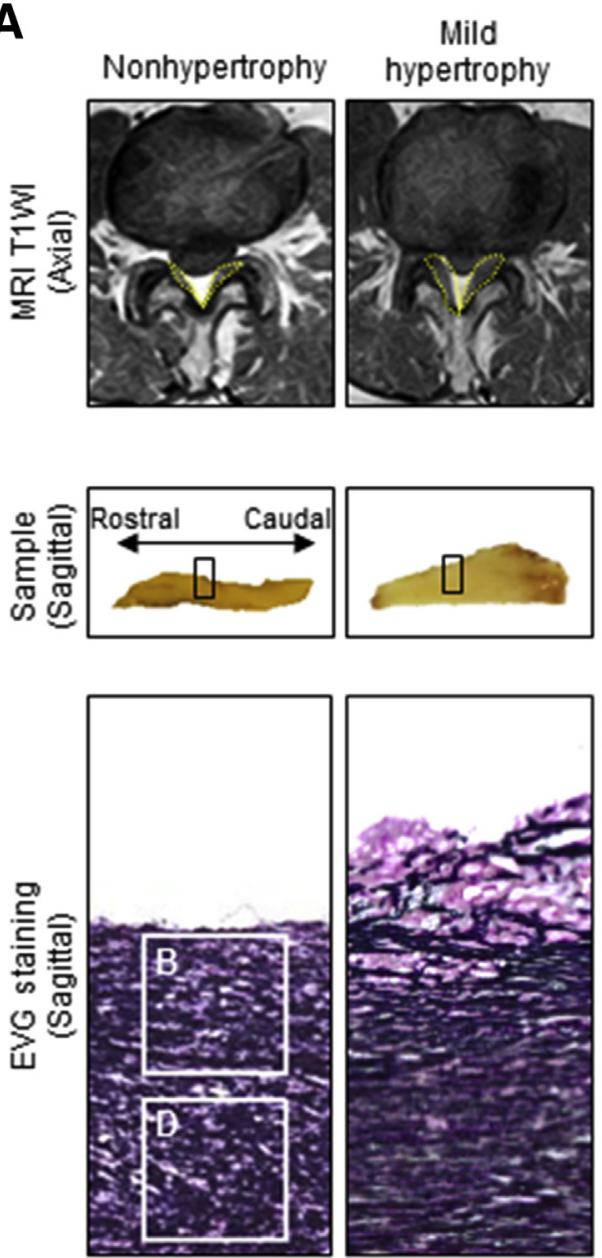

Severe
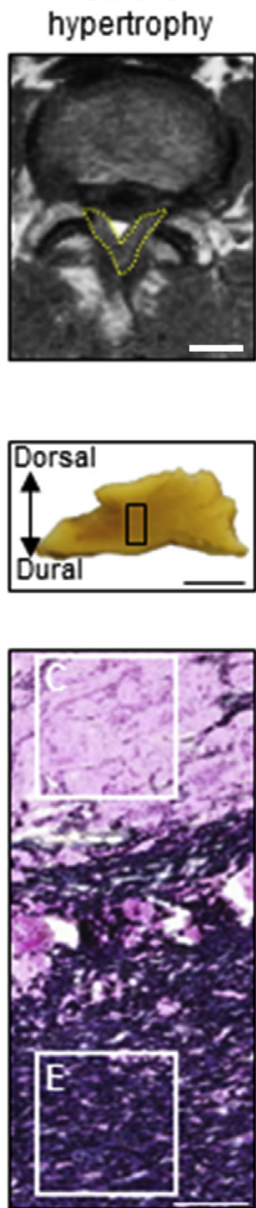

B

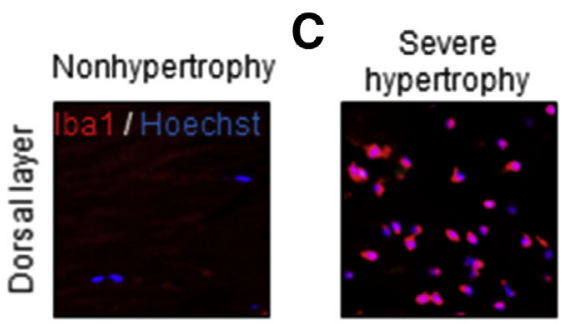

D

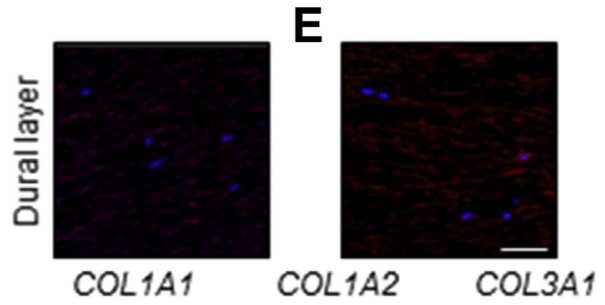

$\mathbf{F}$

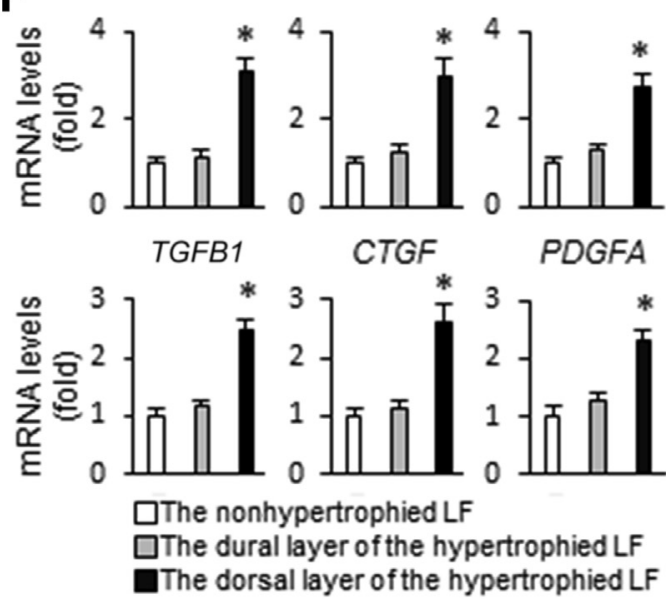

Figure 2 Infiltrating macrophages were associated with the progression of human ligamentum flavum (LF) hypertrophy. A: T1-weighted magnetic resonance imaging (MRI T1WI; upper panels), macroscopic images (middle panels), and Elastica-van Gieson (EVG) staining (lower panels) of human samples: the nonhypertrophied, mildly hypertrophied, and severely hypertrophied LF. The three lower panels are magnifications of the boxed areas in the middle panels. The dashed yellow lines indicate the outline of the LF. B-E: High-magnification views of the boxed areas in the lower panels in A. Immunohistochemical staining of macrophages (Iba1, red) and cell nuclei (Hoechst, blue) in the nonhypertrophied and severely hypertrophied LF. F: The quantitative RT-PCR-based evaluation of the gene expression of collagen and growth factors in the three groups (the nonhypertrophied LF and the dural and dorsal layers of the severely hypertrophied LF). Data are expressed as means \pm SEM (F). ${ }^{*} P<0.05$ (analysis of variance with Tukey-Kramer post hoc test). $n=10$ per group. Scale bars: $2 \mathrm{~cm}$ (A, upper panels); $4 \mathrm{~mm}$ (A, middle panels); $500 \mu \mathrm{m}$ (A, lower panels); $200 \mu \mathrm{m}$ (B-E).

with a sharp 30-gauge needle tip. ${ }^{16}$ Histologic and gene expression analyses were performed at 1,2 , and 6 weeks after microinjury. The control group received sham surgery that only exposed but did not injure the LF ( $n=8$ per group).

To deplete macrophages, clodronate-containing liposomes (clodronate-lip) and control liposomes in phosphate-buffered saline (Katayama Chemical Industries, Osaka, Japan) were injected i.p. (100 $\mu \mathrm{L} / 10 \mathrm{~g}$ body weight, $n=8$ per group) at 0 , $2,4,6,8,10,12$, and 14 days after microinjury (based on their half-life of approximately 48 hours $)^{20}$ without anesthesia.

\section{Laser Microdissection}

At 2 weeks after microinjury, the LFs of COL1a2-GFP transgenic mice were immediately frozen in dry ice/hexane and stored in a deep freezer at $-80^{\circ} \mathrm{C}$ as described previously. ${ }^{21}$ The tissues were cut into 10 - $\mu$ m-thick sections using a cryostat at $-20^{\circ} \mathrm{C}$ and were mounted on polyethylene naphthalate membrane slides. The sections were fixed in ice-cold acetone for 2 minutes. GFP-positive cells were dissected with a laser microdissection (LMD) system (LMD 6500 system; Leica, Tokyo, Japan) and were transferred by gravity into a microcentrifuge tube cap placed directly beneath the section. The tube cap was filled with $75 \mu \mathrm{L}$ of RLT buffer (Qiagen). For each sample, 500 cells were dissected from one series of sections.

\section{Statistical Analysis}

Wilcoxon's rank sum test was used to compare the median values of the data between two groups for the RT-qPCR results after LMD. To analyze the differences among three groups in the LF thickness of the mouse LF with and without microinjury and the RT-qPCR results of human 
A COL1a2-GFP mouse (normal LF, sagittal)
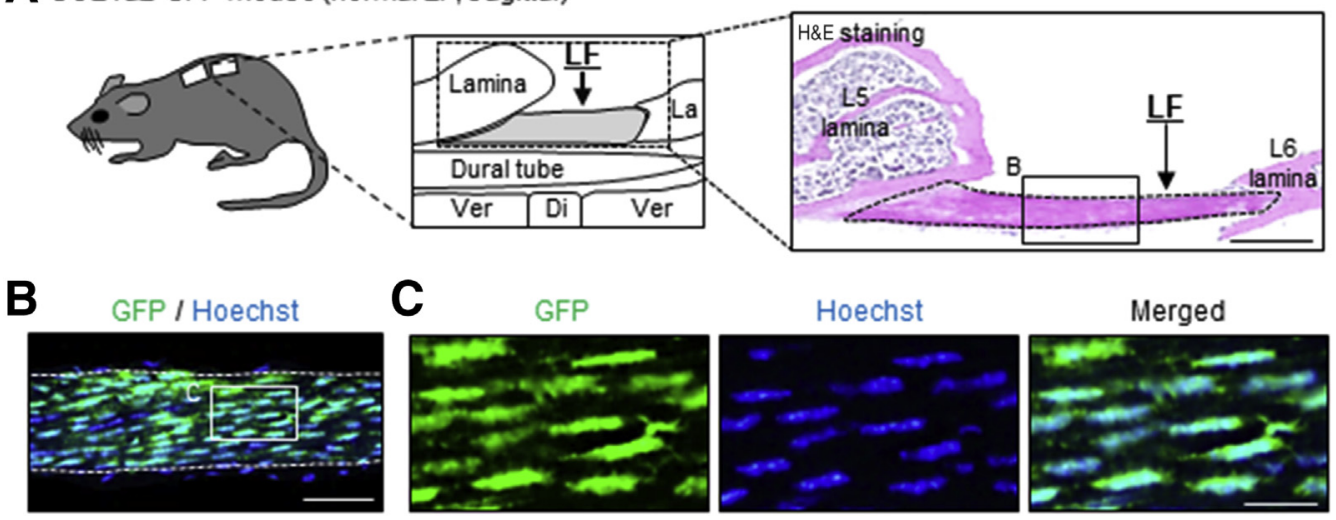

Figure 3 Most of the cells in the normal ligamentum flavum (LF) in collagen type I $\alpha_{2}$ linked to green fluorescent protein (COL1a2-GFP) transgenic mice were GFP-positive fibroblasts. A: Sagittal schematic illustrations and sections of the LF in transgenic reporter mice [hematoxylin and eosin (H\&E) staining]. The heavy dashed black line indicates the outline of the LF. B: Immunohistochemical staining of fibroblasts (GFP, green) and the cell nuclei (Hoechst, blue) in transgenic reporter mice. The dashed white lines indicate the outline of the LF. C: High-magnification views of the boxed area in B. Scale bars: $200 \mu \mathrm{m}$ (A and C); $50 \mu \mathrm{m}$ (B). Di, disk; La, lamina; Ver, vertebral body.

samples and of our microinjured model with and without macrophage depletion, a one-way factorial analysis of variance was performed with the Tukey-Kramer post hoc test. Statistical significance was set at $P<0.05$. The data were presented as means \pm SEM. All statistical analyses were performed using the JMP software program version 11 (SAS Institute Inc., Cary, NC).

\section{Results}

\section{Infiltrating Macrophages Observed in the Human Hypertrophied LF Area}

To investigate the factors associated with LF hypertrophy, we performed a histologic analysis and RT-qPCR of human LF specimens obtained from patients with LSCS and lumbar disk herniation. In the nonhypertrophied LF, EVG staining of the sagittal sections revealed a dense and regular bundle of elastic fibers in the dorsal and dural layers (Figure 2A). In contrast, the severely hypertrophied LF was separated into two distinct layers: the dural layer was mostly composed of rich elastic fibers, whereas the dorsal layer had excessive collagen accumulation without elastic fibers (Figure 2A). In addition, in this dorsal layer, a number of infiltrating Iba1and F4/80-positive macrophages, which have been reported to induce collagen production in fibrotic diseases, ${ }^{11-13}$ were observed (Figure 2, B-E and Supplemental Figure 1A). RT-qPCR revealed that the gene expression of collagen and fibrosis-related factors significantly increased in the dorsal layer of the severely hypertrophied LF compared with the nonhypertrophied LF (Figure 2F). In contrast, in the dural side of the severely hypertrophied LF without macrophage infiltration, the gene expression levels of these factors were similar to the levels in the nonhypertrophied LF. These results suggested that the infiltrating macrophages secreted fibrosis-related factors and contributed to LF hypertrophy.

\section{Fibroblasts in the LF of COL1a2-GFP Transgenic Mice}

It has been difficult to identify fibroblasts in immunohistochemical analyses because of a lack of feasible specific markers. To solve the problem, COL1a2-GFP transgenic mice that express GFP in collagen type I-producing cells have been developed and used for modeling fibrosis and rheumatoid arthritis. ${ }^{15,22,23}$ In the present study, we used the transgenic mice to identify LF fibroblasts and found that most cells in the normal LF were GFP- and vimentin-positive fibroblasts (Figure 3, A-C and Supplemental Figure S2).

\section{Microinjury-Induced Macrophage Infiltration and LF Hypertrophy in the Injured Area}

To examine the influence of macrophages on LF hypertrophy, we induced macrophage infiltration in the dorsal layer of the mouse LF by applying a microinjury. ${ }^{16}$ In the present study, we applied the microinjury selectively in the caudal half of the LF in COL1a2-GFP transgenic mice and left the rostral half intact (Figure 4A). At 6 weeks after microinjury, H\&E staining of the sagittal sections revealed that the LF thickness in the injured area of the microinjury group was significantly increased compared with the sham-operation group (Figure 4, B and C). The LF thickness at the intact area of the microinjury group was comparable with that of the sham-operation group. Notably, collagen accumulation was observed on the dorsal side of the injured area on EVG staining, which was similar to the characteristics observed in the severely hypertrophied LF of human (Figures 2A and 4B). Macrophage infiltration was not observed in immunohistochemical analyses of the LF specimens from the sham-operation group or in the intact area of the microinjury group; however, there were numerous Iba1- and F4/80-positive macrophages in the injured area of the microinjury group at 1 week after 


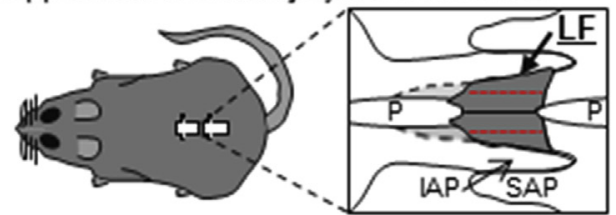

B

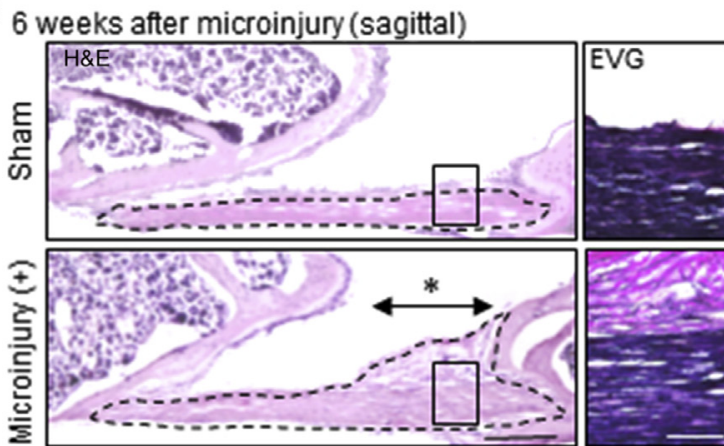

\section{D}
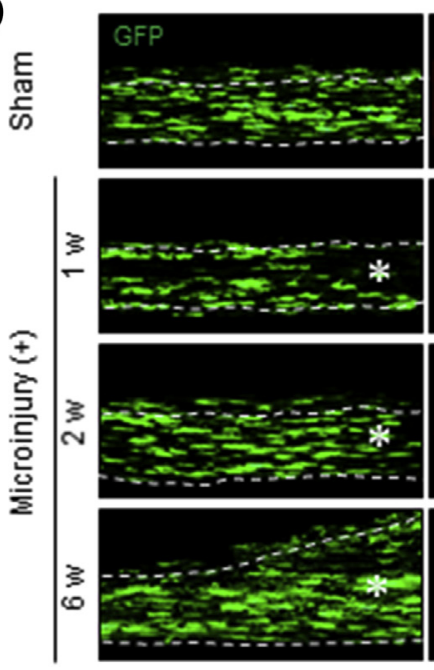

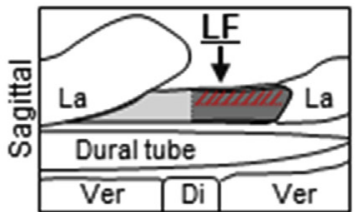

C

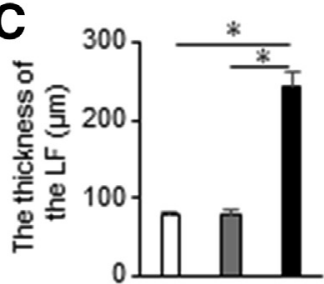

$\square$ The injury (-) LF

$\square$ The rostralhalf of the injury (+) LF

The caudal half of the injury (+) LF
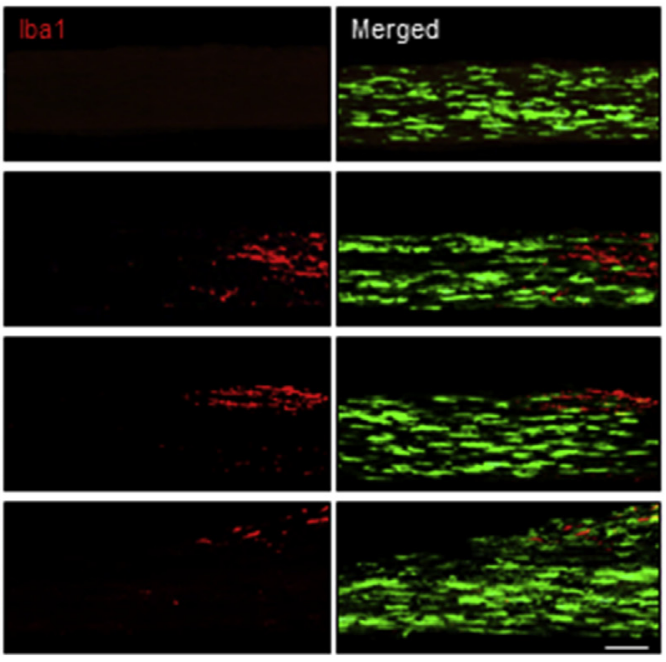

Figure 4 Infiltrating macrophages were involved in ligamentum flavum (LF) hypertrophy in mice. A: Schematic illustrations of the application of a microinjury. The dashed red lines indicate the microinjured area of the LF. B: Sagittal sections of the LF with and without a microinjury on hematoxylin and eosin (H\&E) staining and Elastica-van Gieson (EVG) staining. The asterisk indicates the area of microinjury. The dashed black lines indicate the outline of the LF. C: Bar graphs showing the thickness of the LF in the three groups (the noninjured LF and the rostral half and caudal half of the microinjured LF). D: Immunohistochemical staining of fibroblasts (green fluorescent protein, green), macrophages (Iba1, red), and cell nuclei (Hoechst, blue) in transgenic reporter mice after microinjury. The asterisks indicate the area of microinjury. The dashed white lines indicate the outline of the LF. Data are expressed as means \pm SEM (C). ${ }^{*} P<0.05$ (analysis of variance with Tukey-Kramer post hoc test). $n=8$ per group. Scale bars: $200 \mu \mathrm{m}$ (B, left panels); $50 \mu \mathrm{m}$ (B, right panels, and D). Di, disk; IAP, inferior articular process; La, lamina; $P$, processus spinosus; SAP, superior articular process; Ver, vertebral body. microinjury (Figure 4D and Supplemental Figure 1B). As infiltrating macrophages withdrew, GFP-positive fibroblasts gradually increased in number to fill the injured area and finally banked at the dorsal layer at 6 weeks after microinjury (Figure 4D). These results suggested that infiltrating macrophages were closely associated with LF hypertrophy via collagen production.

\section{Infiltrating Macrophages Stimulated Collagen}

\section{Production in Fibroblasts at the Injure Area}

Because macrophages are unable to produce collagen, we hypothesized that the collagen-producing activity in LF fibroblasts was enhanced in the injured area with macrophage infiltration. To verify this hypothesis, GFP-positive fibroblasts were selectively isolated from the intact and injured areas of the LF in the microinjury group using $\mathrm{LMD},^{21}$ and their profiles were compared (Figure 5A). RT-qPCR revealed that the collagen expression in the fibroblasts in the injured area was significantly higher compared with the intact area (Figure 5B). In addition, the expression of $\alpha$-smooth muscle actin (SMA), which is a marker of myofibroblasts that are closely involved in the pathogenesis of fibrosis via an increased deposition of collagen, ${ }^{24,25}$ significantly increased in the fibroblasts in the injured area (Figure 5B). These findings suggest that the fibroblasts were significantly activated by the infiltrating macrophages, which enhanced their collagen production ability.

\section{Macrophage Depletion Counteracted LF Hypertrophy by} Microinjury

To clarify whether the infiltrating macrophages were crucial for LF hypertrophy, we depleted the circulating macrophages by administering clodronate-lip after creating a microinjury model (Figure 6A). Notably, even at 6 weeks after microinjury, $H \& E$ and EVG staining of the sagittal sections revealed that LF hypertrophy and collagen accumulation were negated when macrophages were depleted (Figure 6, B and C). In 


\section{A Selective isolation of GFP+ fibroblastusing LMD}
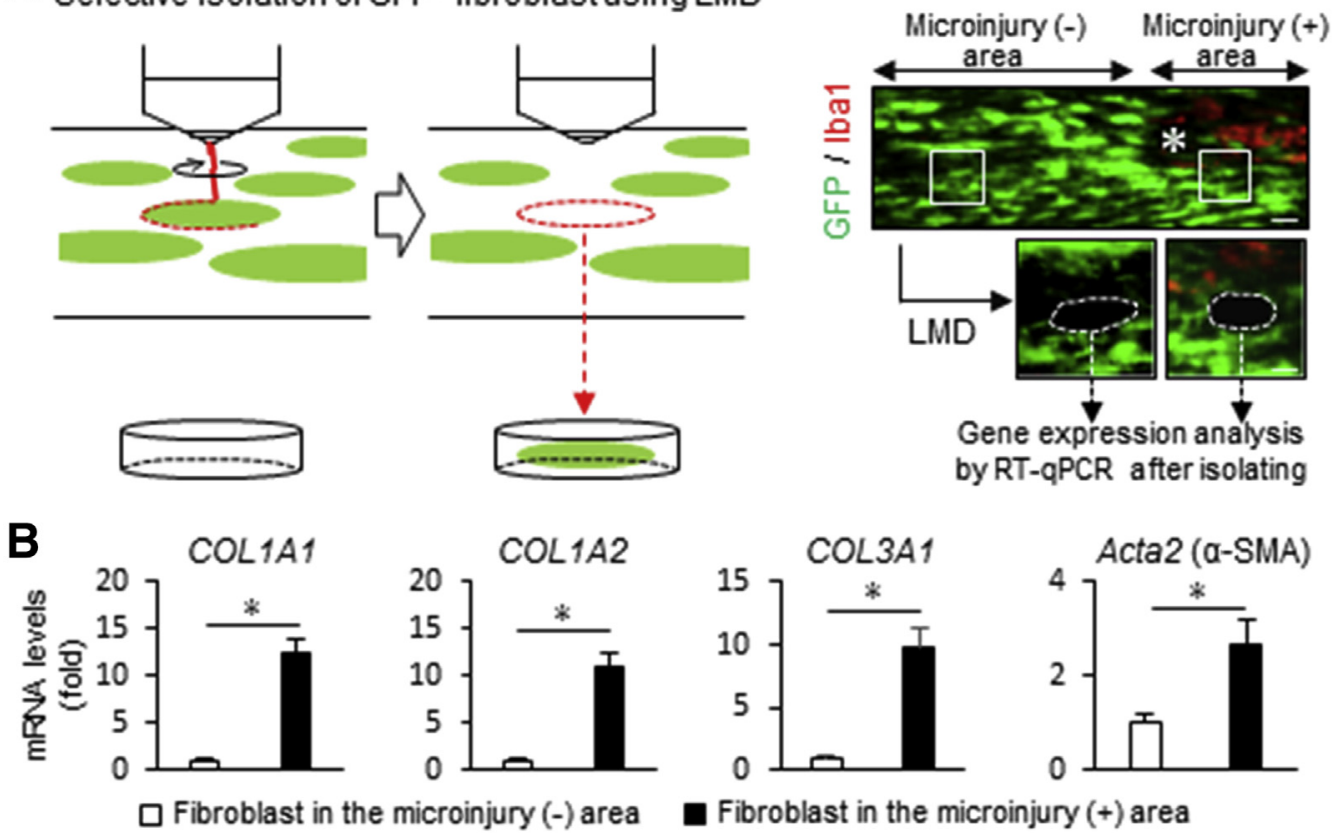

Fibroblast in the microinjury (+) area

Figure 5 The collagen production ability of fibroblasts in the injured area was enhanced. A: Schematic illustrations (left panels) and immunohistochemical images (right panels) showing the selective isolation of green fluorescent protein (GFP)-positive fibroblasts from ligamentum flavum (LF) with microinjury in collagen type I $\alpha_{2}$ linked to GFP (COL1a2-GFP) transgenic mice by laser microdissection (LMD). The asterisk indicates the area of microinjury. The two lower right panels are magnifications of the boxed areas in the upper right panel. The dashed white lines in the lower right panels indicate the outlines of the isolated fibroblasts. B: The quantitative RT-PCR (RT-qPCR)-based evaluation of the gene expression of collagen and $\alpha$-smooth muscle actin (SMA) in the fibroblasts of the two groups after selective isolation (the intact area and injured area of the microinjured LF using LMD). Data are expressed as means \pm SEM (B). ${ }^{*} P<0.05$ (Wilcoxon's rank sum test). $n=8$ per group. Scale bars: $20 \mu \mathrm{m}$ (A, upper right panel); $10 \mu \mathrm{m}$ (A, lower right panels). $\alpha$-SMA, $\alpha$-smooth muscle actin.

addition, in the immunofluorescence analyses, neither macrophage infiltration nor an increase of GFP-positive fibroblasts toward the dorsal layer was observed even at 6 weeks after microinjury (Figure 6D). Furthermore, RT-qPCR revealed that the increased gene expression of Collal, Colla2, Col3al, Tgfbl, Pdgfa, Tnfa, Illb, and Il6 was canceled out in the injured group under macrophage depletion (Figure 7, A-C). These findings strongly suggested that macrophage infiltration was significantly involved in the development of LF hypertrophy via the stimulation of fibroblasts.

\section{Discussion}

In this study, the macrophage depletion experiments with clodronate-lip directly showed that infiltrating macrophages induced LF hypertrophy. In addition, using COL1a2-GFP transgenic mice, we found that the normal LF was mainly composed of fibroblasts and their number increased in the hypertrophied LF. Furthermore, the LMD revealed that the collagen expression was enhanced in fibroblasts adjacent to the infiltrating macrophages in the model of microinjury-induced LF hypertrophy.

The LF has been believed to be mainly composed of fibroblasts based on previous in vitro studies, which reported that spindle-shaped and collagen type I-producing cells were grown after the human LF was minced and cultured. ${ }^{7,10,26}$ However, no studies have yet fully clarified the in vivo cellular composition of the normal LF or the in vivo role of fibroblasts in LF hypertrophy. One of the main reasons for this has been a limitation of selective fibroblast markers. For instance, vimentin is a typical fibroblast marker; however, this also labels other cells, including vascular smooth muscle cells. ${ }^{27}$ The calcium-binding protein S100-A4 and thymus cell antigen- 1 also lack specificity because the proteins are expressed in leukocytes and endothelial cells. ${ }^{28}$ To address the problem, we used COL1a2-GFP transgenic mice in which fibroblasts were labeled as GFP-positive cells, as previously demonstrated in other organ studies. ${ }^{15,22,23}$ By using transgenic reporter mice, quantitative and qualitative analyses of fibroblasts were successfully performed by combining with flow cytometry in the previous study. ${ }^{23}$ In the present study, GFPpositive fibroblasts were selectively isolated by LMD, and the collagen expression in LF fibroblasts varied according to the region with infiltrating macrophages (Figure 5, A and B). These analytical strategies, which combine transgenic reporter mice with selective cell isolation methods, will be useful in studies of fibrotic diseases.

Previous histologic studies using human LF samples reported the fibrotic characteristics, such as the increased collagen fibers, calcification, and ossification, especially at the dorsal layer of the hypertrophied $\mathrm{LF}^{3,29}$ In addition to these, we found the increased expression of fibrosis-related factors, including TGF- $\beta 1$, platelet-derived growth factor, and connective tissue growth factor, in the dorsal layer of the human hypertrophied LF 
A

Experimental protocol

Microinjury

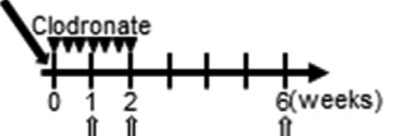

Immunohistological analysis
B

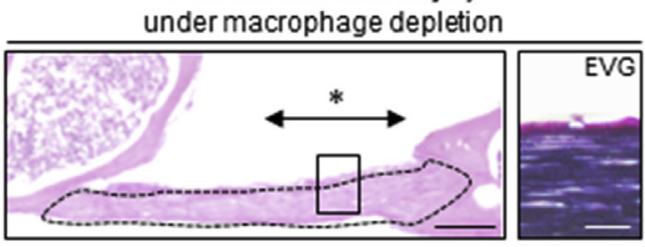

C
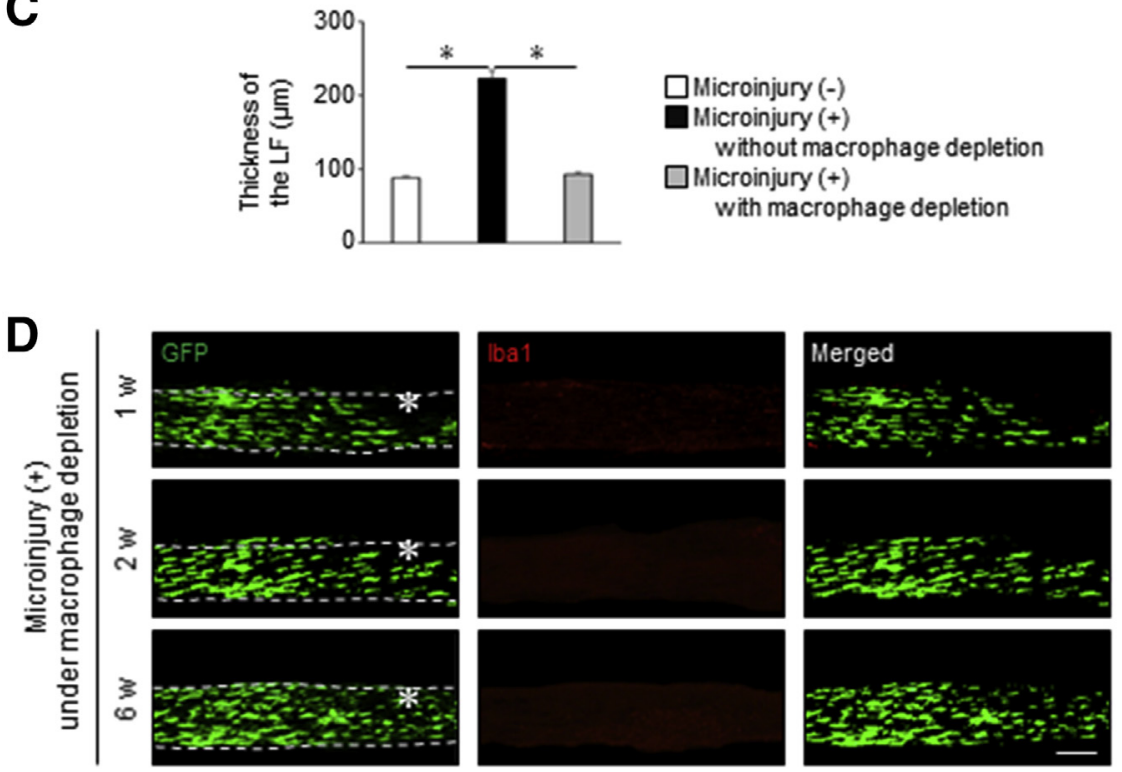

Figure 6 Macrophage depletion attenuated the synthesis of collagen and ligamentum flavum (LF) hypertrophy after microinjury. A: The schedule of clodronate-lip injection for the depletion of macrophages. B: Sagittal sections of the microinjured LF under macrophage depletion [hematoxylin and eosin (H\&E) staining) and Elastica -van Gieson (EVG) staining. The dashed black line indicates the outline of the LF. The asterisk indicates the area of microinjury. The boxed area indicates the dorsal layer of the microinjured LF. The right panel is a magnification of the boxed area in the left panel. C: Bar graphs showing the thickness of the LF in the three groups (the noninjured LF and the microinjured LF with and without macrophage depletion). D: Immunohistochemical staining of fibroblasts [green fluorescent protein (GFP), green], macrophages (Iba1, red), and cell nuclei (Hoechst, blue) in the microinjured LF of collagen type I $\alpha_{2}$ linked to GFP transgenic reporter mice under macrophage depletion. The asterisks indicate the area of microinjury. The dashed white lines indicate the outline of the LF. Data are expressed as means \pm SEM (C). ${ }^{*} P<0.05$ (analysis of variance with Tukey-Kramer post hoc test). $n=8$ per group. Scale bars: $200 \mu \mathrm{m}$ (B, left panel); $50 \mu \mathrm{m}$ (B, right panel, and $\mathbf{D})$.
(Figure 2F). Sairyo et $\mathrm{al}^{29}$ reported that the dorsal layer of the LF was subjected to approximately 1.5 times higher mechanical stress compared with the dural layer during lumbar motion, suggesting that mechanical stress was involved in the fibrotic changes at the dorsal layer. However, although we previously succeeded in establishing a LF hypertrophy model by applying consecutive mechanical stretching stress to the mouse LF, no excessive collagen accumulation was observed at the dorsal
A Collagens

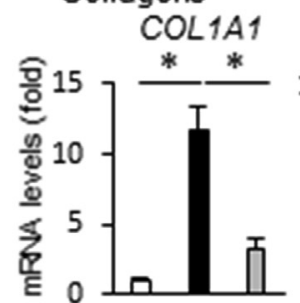

C Inflammatory cytokines

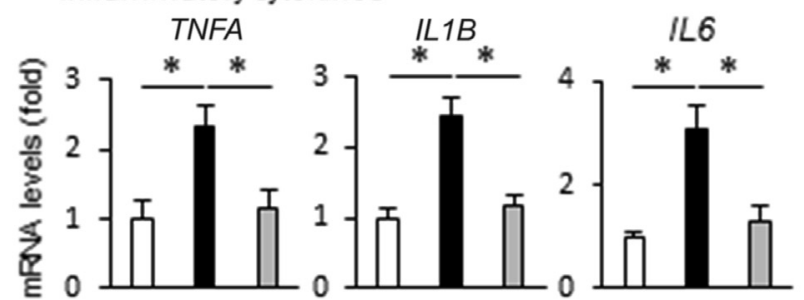

B Fibrogeniccytokines TGFB1

\section{PDGFA}

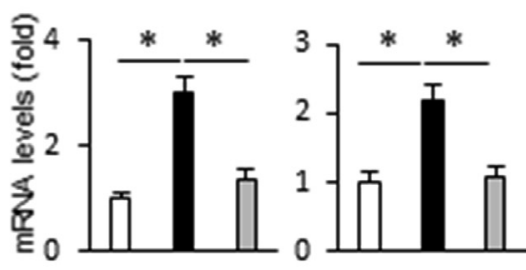

$\square$ Microinjury (-)

Microinjury (+)

without macrophage depletion

$\square$ Microinjury (+)

with macrophage depletion

Figure 7 Macrophage depletion negated the increased expression of collagen and fibrosis-related factors following microinjury. A-C: The quantitative RTPCR-based evaluation of the gene expression of collagen, fibrogenic cytokines, and inflammatory cytokines in the three groups (the noninjured ligamentum flavum and the microinjured ligamentum flavum with and without macrophage depletion). Data are expressed as means \pm SEM. ${ }^{\star} P<0.05$, (analysis of variance with Tukey-Kramer post hoc test. $n=8$ per group. 
layer of the hypertrophied LF. ${ }^{16}$ Thus, we hypothesized that a factor other than mechanical stress was associated with the progression of LF hypertrophy and focused on macrophages, which were suggested to worsen the fibrotic pathology through the secretion of fibroblast-stimulating growth factors in several fibrosis models. ${ }^{11-13}$ In contrast to the previous model of mechanical stress without macrophage infiltration, ${ }^{16}$ we confirmed LF hypertrophy along with collagen accumulation by macrophage infiltration in this study (Figure 4, B-D). We therefore hypothesize that the pathomechanism of LF hypertrophy is biphasic: mechanical stress is significantly involved from the early stage of LF hypertrophy, whereas macrophage infiltration is a trigger for the induction of further hypertrophy via stimulating collagen synthesis.

Macrophages have been reported to contribute to the progression of fibrosis in several organs. ${ }^{11-13}$ They are roughly classified into proinflammatory M1 and anti-inflammatory M2 macrophages, and previous studies have reported that M2 macrophages were important for stimulating collagen synthesis and worsening fibrosis. ${ }^{13,30}$ For example, transferring M2 macrophages, but not M1 macrophages, induced collagen accumulation and worsened renal fibrosis. ${ }^{13,30}$ In CX3CR1-deficient mice in which the infiltration of M2 macrophages was inhibited, skin fibrotic lesions were significantly suppressed compared with wild-type mice. ${ }^{31}$ Considering that a histologic characteristic of LF hypertrophy is also abnormal collagen deposition (Figure 1), we speculate that M2 macrophages play an important role in LF hypertrophy.

TGF- $\beta 1$ has been considered a particularly important factor for stimulating collagen synthesis in fibrotic diseases of several organs. ${ }^{32,33}$ In addition, this cytokine also promotes the differentiation of fibroblasts to myofibroblasts. ${ }^{34-36}$ Myofibroblasts are known to express $\alpha$-SMA and contribute to the development of fibrosis by producing abnormal collagen. ${ }^{24,25}$ In the human hypertrophied LF, $\alpha$-SMA-positive myofibroblasts were reported to be observed. ${ }^{37}$ Hur et $\mathrm{al}^{37}$ reported that TGF- $\beta 1$ increased the gene expression of $\alpha$-SMA and collagen in cultured human LF cells. These results suggested that TGF- $\beta 1$ was associated with LF hypertrophy through the promotion of collagen production and myofibroblast differentiation. In the present study, we found that the increased expression of TGF- $\beta 1$ induced by microinjury was significantly suppressed in our macrophage depletion model (Figures 5 and 7), strongly indicating that the main source of this cytokine in LF hypertrophy was infiltrating macrophages. Therefore, we suggest that infiltrating macrophages are crucial for the progression of LF hypertrophy via TGF- $\beta 1$ secretion associated with collagen production and myofibroblast differentiation.

The present study has several limitations. The origin of the increase in fibroblasts in the hypertrophied LF remains unclear. Fibroblasts are largely classified into two types: interstitial resident fibroblasts and bone marrow-derived fibroblasts (ie, circulating fibrocytes). It is controversial which type of fibroblasts deeply contribute to collagen deposition in fibrotic diseases. ${ }^{15,23,38}$ Further studies are needed to determine whether the proliferation of resident fibroblasts or the infiltration of circulating fibrocytes is mainly involved in LF hypertrophy to clarify the nonsurgical therapeutic targets in LF hypertrophy. In addition to this limitation, it is not clear whether the accumulation of mechanical stress indeed causes microinjury at the dorsal layer of the human LF. However, we believe that our model of LF hypertrophy with macrophage infiltration reveals the pathologic characteristics of human LF hypertrophy and is therefore useful for obtaining a better understanding of its pathogenesis.

In conclusion, we showed, for the first time, that macrophage infiltration was a causative factor for LF hypertrophy via collagen accumulation in mice. In addition, we showed in vivo that fibroblasts were a source of collagen production in LF hypertrophy using the COL1a2-GFP transgenic mice. Furthermore, the experiments using LMD revealed that infiltrating macrophages were associated with LF hypertrophy via activating fibroblasts.

\section{Acknowledgments}

T.S. designed and performed most of the experiments with technical help from M.Har., H.K., K.Ko., K.Y., K.Ki., and S.Y.; K.H., Y.M., K.Ka., M.Hay., Y.I., K.S., and Y.N. provided experimental support and ideas for the project; S.O. designed the studies, supervised the overall project, and performed the final manuscript preparation.

\section{Supplemental Data}

Supplemental material for this article can be found at https://doi.org/10.1016/j.ajpath.2017.08.020.

\section{References}

1. Szpalski M, Gunzburg R: Lumbar spinal stenosis in the elderly: an overview. Eur Spine J 2003, 12 Suppl 2:S170-S175

2. Towne EB, Reichert FL: Compression of the lumbosacral roots of the spinal cord by thickened ligamenta flava. Ann Surg 1931, 94:327-336

3. Schräder PK, Grob D, Rahn BA, Cordey J, Dvorak J: Histology of the ligamentum flavum in patients with degenerative lumbar spinal stenosis. Eur Spine J 1999, 8:323-328

4. Yoshida M, Shima K, Taniguchi Y, Tamaki T, Tanaka T: Hypertrophied ligamentum flavum in lumbar spinal canal stenosis: pathogenesis and morphologic and immunohistochemical observation. Spine 1992, $17: 1353-1360$

5. Löhr M, Hampl JA, Lee JY, Ernestus RI, Deckert M, Stenzel W: Hypertrophy of the lumbar ligamentum flavum is associated with inflammationrelated TGF- $\beta$ expression. Acta Neurochir 2011, 153:134-141

6. Nakatani T, Marui T, Hitora T, Doita M, Nishida K, Kurosaka M: Mechanical stretching force promotes collagen synthesis by cultured cells from human ligamentum flavum via transforming growth factorbeta1. J Orthop Res 2002, 20:1380-1386

7. Zhong ZM, Zha DS, Xiao WD, Wu SH, Wu Q, Zhang Y, Liu FQ, Chen JT: Hypertrophy of ligamentum flavum in lumbar spine stenosis associated with the increased expression of connective tissue growth factor. J Orthop Res 2011, 29:1592-1597

8. Bataller R, Brenner DA: Liver fibrosis. J Clin Invest 2005, 115: 209-218 
9. Wynn TA, Ramalingam TR: Mechanisms of fibrosis: therapeutic translation for fibrotic disease. Nat Med 2012, 18:1028-1040

10. Specchia N, Pagnotta A, Gigante A, Logroscino G, Toesca A: Characterization of cultured human ligamentum flavum cells in lumbar spine stenosis. J Orthop Res 2001, 19:294-300

11. Schneider DJ, Wu M, Le TT, Cho SH, Brenner MB, Blackburn MR, Agarwal SK: Cadherin-11 contributes to pulmonary fibrosis: potential role in TGF- $\beta$ production and epithelial to mesenchymal transition. FASEB J 2012, 26:503-512

12. Chu PS, Nakamoto N, Ebinuma H, Usui S, Saeki K, Matsumoto A, Mikami Y, Sugiyama K, Tomita K, Kanai T, Saito H, Hibi T: C-C motif chemokine receptor 9 positive macrophages activate hepatic stellate cells and promote liver fibrosis in mice. Hepatology 2013, 58:337-350

13. Kim MG, Kim SC, Ko YS, Lee HY, Jo SK, Cho W: The role of M2 macrophages in the progression of chronic kidney disease following acute kidney injury. PLoS One 2015, 10:e0143961

14. Altinkaya N, Yildirim T, Demir S, Alkan O, Sarica FB: Factors associated with the thickness of the ligamentum flavum: is ligamentum flavum thickening due to hypertrophy or buckling? Spine 2011, 36: E1093-E1097

15. Higashiyama R, Moro T, Nakao S, Mikami K, Fukumitsu H, Ueda Y, Ikeda K, Adachi E, Bou-Gharios G, Okazaki I, Inagaki Y: Negligible contribution of bone marrow-derived cells to collagen production during hepatic fibrogenesis in mice. Gastroenterology 2009, 137:1459-1466.e1

16. Saito T, Yokota K, Kobayakawa K, Hara M, Kubota K, Harimaya K, Kawaguchi K, Hayashida M, Matsumoto Y, Doi T, Shiba K. Nakashima Y, Okada S: Experimental mouse model of lumbar ligamentum flavum hypertrophy. PLoS One 2017, 12:e0169717

17. Kirihara Y, Takechi M, Kurosaki K, Kobayashi Y, Kurosawa T: Anesthetic effects of a mixture of medetomidine, midazolam and butorphanol in two strains of mice. Exp Anim 2013, 62:173-180

18. Kumamaru H, Saiwai H, Ohkawa Y, Yamada H, Iwamoto Y, Okada S: Age-related differences in cellular and molecular profiles of inflammatory responses after spinal cord injury. J Cell Physiol 2012, 227:1335-1346

19. Zhang Y, Chen J, Zhong ZM, Yang D, Zhu Q: Is platelet-derived growth factor-BB expression proportional to fibrosis in the hypertrophied lumber ligamentum flavum? Spine 2010, 35:E1479-E1486

20. Tacke F, Ginhoux F, Jakubzick C, van Rooijen N, Merad M, Randolph GJ: Immature monocytes acquire antigens from other cells in the bone marrow and present them to $\mathrm{T}$ cells after maturing in the periphery. J Exp Med 2006, 203:583-597

21. Yokota K, Kobayakawa K, Kubota K, Miyawaki A, Okano H, Ohkawa Y, Iwamoto Y, Okada S: Engrafted neural stem/progenitor cells promote functional recovery through synapse reorganization with spared host neurons after spinal cord injury. Stem Cell Reports 2015, 5:264-277

22. Matsuo Y, Mizoguchi F, Saito T, Kawahata K, Ueha S, Matsushima K, Inagaki Y, Miyasaka N, Kohsaka H: Local fibroblast proliferation but not influx is responsible for synovial hyperplasia in a murine model of rheumatoid arthritis. Biochem Biophys Res Commun 2016, 470:504-509

23. Tsukui T, Ueha S, Abe J, Hashimoto S, Shichino S, Shimaoka T, Shand FH, Arakawa Y, Oshima K, Hattori M, Inagaki Y, Tomura M, Matsushima K: Qualitative rather than quantitative changes are hallmarks of fibroblasts in bleomycin-induced pulmonary fibrosis. Am J Pathol 2013, 183:758-773
24. Gabbiani G: The myofibroblast in wound healing and fibrocontractive diseases. J Pathol 2003, 200:500-503

25. Darby IA, Zakuan N, Billet F, Desmoulière A: The myofibroblast, a key cell in normal and pathological tissue repair. Cell Mol Life Sci 2016, 73:1145-1157

26. Cao YL, Duan Y, Zhu LX, Zhan YN, Min SX, Jin AM: TGF- $\beta 1$, in association with the increased expression of connective tissue growth factor, induce the hypertrophy of the ligamentum flavum through the p38 MAPK pathway. Int J Mol Med 2016, 38:391-398

27. Zeisberg EM, Kalluri R: Origins of cardiac fibroblasts. Circ Res 2010, 107:1304-1312

28. Zeisberg EM, Tarnavski O, Zeisberg M, Dorfman AL, McMullen JR, Gustafsson E, Chandraker A, Yuan X, Pu WT, Roberts AB, Neilson EG, Sayegh MH, Izumo S, Kalluri R: Endothelial-tomesenchymal transition contributes to cardiac fibrosis. Nat Med 2007, 13:952-961

29. Sairyo K, Biyani A, Goel V, Leaman D, Booth R Jr, Thomas J, Gehling D, Vishnubhotla L, Long R, Ebraheim N: Pathomechanism of ligamentum flavum hypertrophy: a multidisciplinary investigation based on clinical, biomechanical, histologic, and biologic assessments. Spine 2005, 30:2649-2656

30. Pan B, Liu G, Jiang Z, Zheng D: Regulation of renal fibrosis by macrophage polarization. Cell Physiol Biochem 2015, 35:1062-1069

31. Arai M, Ikawa Y, Chujo S, Hamaguchi Y, Ishida W, Shirasaki F, Hasegawa M, Mukaida N, Fujimoto M, Takehara K: Chemokine receptors CCR2 and CX3CR1 regulate skin fibrosis in the mouse model of cytokine-induced systemic sclerosis. J Dermatol Sci 2013, 69: $250-258$

32. Li J, Campanale NV, Liang RJ, Deane JA, Bertram JF, Ricardo SD: Inhibition of p38 mitogen-activated protein kinase and transforming growth factor-beta1/Smad signaling pathways modulates the development of fibrosis in Adriamycin-induced nephropathy. Am J Pathol 2006, 169:1527-1540

33. Li J, Chen J, Kirsner R: Pathophysiology of acute wound healing. Clin Dermatol 2007, 25:9-18

34. Vyas B, Ishikawa K, Duflo S, Chen X, Thibeault SL: Inhibitory effects of hepatocyte growth factor and interleukin-6 on transforming growth factor-beta1 mediated vocal fold fibroblast-myofibroblast differentiation. Ann Otol Rhinol Laryngol 2010, 119:350-357

35. Guo W, Shan B, Klingsberg RC, Qin X, Lasky JA: Abrogation of TGF-beta1-induced fibroblast-myofibroblast differentiation by histone deacetylase inhibition. Am J Physiol Lung Cell Mol Physiol 2009, 297:L864-L870

36. Hu B, Wu Z, Phan SH: Smad3 mediates transforming growth factorbeta-induced alpha-smooth muscle actin expression. Am J Respir Cell Mol Biol 2003, 29:397-404

37. Hur JW, Bae T, Ye S, Kim JH, Lee S, Kim K, Lee SH, Kim JS, Lee JB, Cho TH, Park JY, Hur JK: Myofibroblast in the ligamentum flavum hypertrophic activity. Eur Spine J 2017, 26:2021-2030

38. Tanaka M, Ikeda K, Suganami T, Komiya C, Ochi K, Shirakawa I, Hamaguchi M, Nishimura S, Manabe I, Matsuda T, Kimura K, Inoue $\mathrm{H}$, Inagaki $\mathrm{Y}$, Aoe $\mathrm{S}$, Yamasaki S, Ogawa Y: Macrophageinducible C-type lectin underlies obesity-induced adipose tissue fibrosis. Nat Commun 2014, 5:4982 